\section{Ausschreibungen}

Wahlen im Rahmen der Mitgliederversammlung am 28. September 2013

Anlässlich des kommenden Bundeskongresses in Leipzig im September 2013 stehen Vorstandswahlen an. Wer an einer der folgenden Aufgaben interessiert ist oder gewählt ist und bereit wäre, die Tätigkeit fortzusetzen, wird gebeten, dies der djb-Bundesgeschäftsstelle mitzuteilen. Auch Vorschläge für Kandidaturen sind willkommen. Bewerberinnen haben die Möglichkeit, sich in der Ausgabe der djbZ 3/2013 (Redaktionsschluss: 15. Juni 2013) kurz vorzustellen. Wir freuen uns über Bewerbungen mit Kurzporträt und Angaben zu Arbeitsschwerpunkten und geplanter Sacharbeit. Eine Bewerbung ist auch im Rahmen der Mitgliederversammlung möglich.

\section{Präsidium}

Nach $\mathbb{} 7$ der djb-Bundessatzung ist das Präsidium alle zwei Jahre von der Mitgliederversammlung zu wählen. Die derzeitigen Präsidiumsmitglieder (Präsidentin Ramona Pisal, Vizepräsidentinnen Margarete Hofmann und Eva Schübel) beabsichtigen, erneut zu kandidieren. Schatzmeisterin Dagmar Brinkmann stellt ihr Amt nach nunmehr achtjähriger Amtszeit zur Verfügung.

\section{Vorsitzende der Ständigen Kommissionen}

Nach Inkrafttreten der aktuellen Satzung wurden im Jahr 2001 erstmalig Kommissionsvorsitzende in den Bundesvorstand gewählt. Die vierjährige Amtszeit der Vorsitzenden der jetzigen fünf Ständigen Kommissionen endet im September 2013. Zu wählen sind die Vorsitzenden der:

- Kommission Arbeits-, Gleichstellungs- und Wirtschaftsrecht

- Kommission Zivil-, Familien- und Erbrecht, Recht anderer Lebensgemeinschaften

- Kommission Strafrecht

- Kommission Recht der sozialen Sicherung, Familienlastenausgleich

- Kommission Öffentliches Recht, Europa- und Völkerrecht Die Kandidaturen sind nicht auf bisherige Kommissionsmitglieder beschränkt.

\section{Beisitzerin(nen)}

Schließlich ist nach $\$ 6$ Absatz 4 der Bundessatzung die Position von bis zu zwei Beisitzerinnen neu zu besetzen, von denen (mindestens) eine die Vertreterin der Mitglieder in Ausbildung ist.

\section{djb-Delegierte}

Am 28. September 2013 wird die Mitgliederversammlung auch Vertreterinnen des djb in Vereinen wählen, in denen dieser Mitglied ist. Folgende Posten sind zu vergeben:

- Deutsche Liga für das Kind

- European Women Lawyers Association (EWLA)

- UN Women Deutschland (vormals UNIFEM)

- Deutsche Stiftung für internationale rechtliche Zusammenarbeit (IRZ-Stiftung)

- National Coalition für die Umsetzung der UN-Kinderrechtskonvention in Deutschland (NC)

- Netzwerk Europäische Bewegung Deutschland (EBD)

\section{Mitarbeit in den Kommissionen}

Die Amtszeit der Mitglieder für die derzeit bestehenden fünf Ständigen Kommissionen läuft am 28. September 2013 ab. Das sind die Kommissionen:

- Arbeits-, Gleichstellungs- und Wirtschaftsrecht

- Zivil-, Familien- und Erbrecht, Recht anderer Lebensgemeinschaften

- Strafrecht

- Recht der sozialen Sicherung, Familienlastenausgleich

- Öffentliches Recht, Europa- und Völkerrecht

Kolleginnen, die als Mitglied in der kommenden Wahlperiode in einer dieser Kommissionen mitarbeiten möchten, bitten wir, sich für die Mitgliedschaft in der gewünschten Kommission schriftlich bis zum 13. Oktober 2013 über die Geschäftsstelle beim Bundesvorstand zu bewerben. Die Bewerbung sollte in kurzer Form Angaben zur spezifischen Kompetenz und Erfahrung auf dem jeweiligen Arbeitsgebiet enthalten. Bitte geben Sie auch an, für welchen Arbeitsbereich des Rechtsgebietes Sie sich interessieren. Auch diejenigen, die schon bisher in einer der Kommissionen mitgearbeitet haben, werden gebeten, ihre Arbeitsbereitschaft erneut der Geschäftsstelle mitzuteilen.

Die Mitglieder dieser Ständigen Kommissionen werden während der ersten ordentlichen Bundesvorstandssitzung nach der Wahl auf Vorschlag der Kommissionsvorsitzenden vom Bundesvorstand bestellt und anschließend über die Entscheidung informiert. Weitere Informationen zur Kommissionsarbeit finden Sie in der Geschäftsordnung für Kommissionen und Arbeitsstäbe des djb, abrufbar im Mitgliederbereich auf unserer Homepage (http://www.djb.de/ Kommissionen/Kommissionsarbeit/).

\title{
Gründung der djb-Regionalgruppe Madrid
}

Nach mehreren informellen Treffen seit dem letzten Sommer wurde am 23. April 2013 die Regionalgruppe Madrid im djb offiziell gegründet. Zur Vorsitzenden wurde Katharina Miller, LL.M. gewählt, Elisabeth Tesch als ihre Stellvertreterin und Isabel Stockton als Schatzmeisterin.
Neben der Regionalgruppe Brüssel ist dies die zweite ausländische Gruppe des djb mit monatlichen Treffen. Für Fragen, Anmerkungen und Kontaktaufnahme können Sie sich gerne mit Katharina Miller (katharina-miller@gmx.de) in Verbindung setzen. 